\title{
Predictors of hospital mortality among septic ICU patients with Acinetobacter spp. bacteremia: a cohort study
}

\author{
Andrew F Shorr ${ }^{1}$, Marya D Zilberberg ${ }^{2,3^{*}}$, Scott T Micek ${ }^{4}$ and Marin H Kollef ${ }^{5,6}$
}

\begin{abstract}
Background: We hypothesized that among septic ICU patients with Acinetobacter spp. bacteremia (Ac-BSI), carbapenem-resistant Acinetobacter spp. (CRAc) increase risk for inappropriate initial antibiotic therapy (non-IAAT), and non-IAAT is a predictor of hospital death.

Methods: We conducted a retrospective cohort study of adult septic ICU patients with Ac-BSI. Non-IAAT was defined as exposure to initially prescribed antibiotics not active against the pathogen based on in vitro susceptibility testing, and having no exposure to appropriate antimicrobial treatment within 24 hours of drawing positive culture. We compared patients who died to those who survived, and derived regression models to identify predictors of hospital mortality and of non-IAAT.
\end{abstract}

Results: Out of 131 patients with Ac-BSI, 65 (49.6\%) died (non-survivors, NS). NS were older $(63$ [51, 76] vs. 56 [45, 66] years, $p=0.014)$, and sicker than survivors (S): APACHE II $(24[19,31]$ vs. $18[13,22], p<0.001)$ and Charlson $(5[2,8]$ vs. 3 $[1,6], p=0.009)$ scores. NS were also more likely than $S$ to require pressors $(75.4 \%$ vs. $42.4 \%, p<0.001)$ and mechanical ventilation (75.4\% vs. $53.0 \%, p=0.008)$. Both CRAc (69.2\% vs. $47.0 \%, p=0.010)$ and non-IAAT ( $83.1 \%$ vs. $59.1 \%, p=0.002)$ were more frequent among NS than S. In multivariate analyses, non-IAAT emerged as an independent predictor of hospital death (risk ratio [RR] 1.42, 95\% confidence interval [CI] 1.10-1.58), while CRAc was the single strongest predictor of non-IAAT (RR 2.66, 95\% Cl 2.43-2.72).

Conclusions: Among septic ICU patients with Ac-BSI, non-IAAT predicts mortality. Carbapenem resistance appears to mediate the relationship between non-IAAT and mortality.

Keywords: Aevere sepsis, Acinetobacter spp, Carbapenem resistance, Inappropriate initial antibiotic therapy, Mortality, Bacteremia

\section{Background}

In its 2013 report titled "Antibiotic Resistance Threats in the United States, 2013", the US Centers for Disease Control and Prevention rated carbapenem resistance as an urgent (among Enterobacteriaceae) or serious (among Acinetobacter spp.) threat [1]. Since anti-pseudomonal carbapenems often represent a last-resort option, emerging resistance to this class indeed reflects a grave concern. The growing prevalence of carbapenem-resistant pathogens makes it difficult for the clinician to choose reliably

\footnotetext{
* Correspondence: evimedgroup@gmail.com

2EviMed Research Group, LLC, Goshen, MA, USA

${ }^{3}$ School of Public Health and Health Sciences, University of Massachusetts, Amherst, MA, USA

Full list of author information is available at the end of the article
}

what will qualify as initially appropriate antibiotic therapy (IAAT).

Understandably, of greatest concern is carbapenem resistance among Enterobacteriaceae and Pseudomonas aeruginosa, as they typically comprise the dominant organisms encoding carbapenemases [2-5]. Acinetobacter spp., on the other hand, is a less frequently encountered pathogen. However, the proportion of carbapenem resistance among Acinetobacter spp. isolates is $2-5$ times that seen in Pseudomonas aeruginosa and Klebsiella spp., and over an order of magnitude that among E. coli [3].

In the setting of many serious infections, one of the critical determinants of the outcome is early empiric coverage for the culprit organism [6-14]. Failing to administer in a 
timely manner an agent that is in vitro active against the offending pathogen(s) leads to increased mortality and morbidity. Acknowledgement of this observation has led to the practice of de-escalation where one often starts with an initially broad anti-infective (or a combination of agents) and later narrows the spectrum of coverage when culture results become available [15]. Unfortunately, for Acinetobacter spp. it is unclear if and how one can successfully execute this strategy, particularly in light of the fact that most isolates are resistant to last-resort treatment. Similarly, as a matter of health policy and antibiotic stewardship, it is necessary to appreciate the contribution of carbapenem resistance to the poor outcomes in infections with Acinetobacter spp. Gaining insight into the relationship between resistance and outcomes specifically as it relates to this pathogen may help clarify the urgency of the need for novel agents with which to treat it.

We hypothesized that in the setting of severe sepsis or septic shock due to Acinetobacter spp., failure to receive IAAT increased the risk of death, and that, as a corollary, carbapenem resistance is a strong determinant of exposure to non-IAAT.

\section{Methods}

\section{Study design and ethical standards}

We conducted a single-center retrospective cohort study from January 2002 to December 2012. Barnes-Jewish Hospital is a 1,200-bed urban academic medical center located in St. Louis, MO. The study was approved by the Washington University School of Medicine Human Studies Committee and informed consent was waived.

\section{Study cohort}

All consecutive adult ICU patients between January 2002 and December 2012 were enrolled if 1). They had a positive blood culture for Acinetobacter spp., and 2). There was an International Classification of Diseases, version 9, clinical modification (ICD-9-CM) code for an acute organ dysfunction [16]. Only the first episode of sepsis was included.

\section{Definitions}

To be included in the analysis patients had to meet criteria for severe sepsis based on discharge ICD-9-CM codes for acute organ dysfunction [16]. Septic shock was present if vasopressors (norepinephrine, dopamine, epinephrine, phenylephrine or vasopressin) were initiated within 24 hours of the blood culture collection date and time. Antimicrobial treatment was deemed IAAT if the initially prescribed antibiotic regimen was active against the identified pathogen based on in vitro susceptibility testing and administered within 24 hours following blood culture collection; all other regimens were classified as non-IAAT. Because the role of combination therapy in treating Acinetobacter spp. is not well defined, combination therapy was not a criterion for defining IAAT. We also required that antibiotics be prescribed for $\geq 24$ hours. Prior antibiotic exposure occurred within the preceding 90 days, as did prior hospitalization, while prior bacteremia was defined within 30 days of the current episode. The bacteremia was deemed to be a healthcareassociated complication (HAC) if one of the following risk factors was identified: 1). Need for dialysis; 2). Immune suppression; 3). Prior hospitalization; 4). Prior antibiotics; 5). Current infection deemed hospital-acquired blood stream infection (HABSI; onset of infection $\geq 2$ days after admission). Carbapenem-resistant Acinetobacter spp. (CRAc) was present if it was the organism that grew out in at least one blood culture specimen.

\section{Antimicrobial susceptibility testing}

The microbiology laboratory performed antimicrobial susceptibility of the isolates using the disk diffusion method according to guidelines and breakpoints established by the Clinical Laboratory and Standards Institute (CLSI) appropriate to the year the organism was isolated.

\section{Data elements}

Patient baseline characteristics and process of care variables were collected from the automated hospital medical record, microbiology database, and pharmacy database of Barnes-Jewish Hospital. Electronic inpatient and outpatient medical records available for all patients in the BJC Healthcare system were reviewed to determine prior antibiotic exposure. The baseline characteristics collected included: age, gender, race, past history of congestive heart failure, chronic obstructive pulmonary disease, diabetes mellitus, chronic liver disease, underlying malignancy, and end-stage renal disease requiring dialysis. The Acute Physiology and Chronic Health Evaluation (APACHE) II and Charlson comorbidity scores were calculated based on clinical data present during the twenty-four hours after the positive blood cultures were obtained [17]. This was done to accommodate patients with community-acquired and healthcare-associated community-onset infections who only had clinical data available after blood cultures were drawn. The primary outcome variable was hospital mortality. Because we were interested in understanding the contribution of CRAc to the risk of receiving nonIAAT, we examined it as a secondary endpoint in a logistic regression.

\section{Statistical analyses}

Continuous variables were reported as means with standard deviations when distributed normally, or medians with 25th and 75th percentiles when skewed. Differences between mean values were tested via Student's $t$-test, while those between medians were examined using the Mann-Whitney 
$U$ test. Categorical data were summarized as proportions, and the Chi-square test or Fisher's exact test for small samples was used to examine differences between groups. We developed several multiple logistic regression models to identify clinical risk factors associated with hospital mortality. In the mortality models, all risk factors that were significant at $\leq 0.20$ in the univariate analyses, as well as all biologically plausible factors even if they did not reach this level of significance, were included in the corresponding multivariable analyses. All variables entered into the models were examined to assess for co-linearity, and interaction terms were tested. The most parsimonious models were derived using the backward manual elimination method, and the best-fitting model was chosen based on the c-statistic. The model's calibration was assessed with the HosmerLemeshow goodness-of-fit test. To exclude the influence of time-dependent covariates on hospital mortality, we confirmed the risk factors in a Cox proportional hazards model. Similarly, the most parsimonious model for the predictors of non-IAAT was computed and its fit was tested with the c-statistic and the Hosmer-Lemeshow goodnessof-fit test.

Because both outcomes of interest in each of the models (mortality and non-IAAT) occurred with high frequency (overall mortality $49.6 \%$, overall non-IAAT $71.0 \%$ ), the adjusted odds ratios would overestimate the magnitude of the actual risk associated with each of the independent variables examined. For this reason we corrected the risk estimate according to the method of Zhang [18], and report the corrected risk ratios.

All tests were two-tailed, and a $\mathrm{p}$ value $<0.05$ was deemed a priori to represent statistical significance. All calculations were done in Stata/SE, version 9 (StataCorp, College Station, TX).

\section{Results}

One hundred and thirty-one patients with severe sepsis or septic shock due to Acinetobacter spp. met the inclusion criteria. Among these 76 (58.0\%) were CRAc. (Table 1 lists additional drug susceptibilities stratified by carbapenem resistance). Overall hospital mortality was $49.6 \%$. The patients' baseline characteristics are listed in Table 2. Those who died were older and had a higher comorbidity burden, as signified by the Charlson comorbidity score, than those who survived their hospitalization. A higher proportion of those patients who died prior to discharge had dialysis $(28.1 \%$ vs. $15.2 \%, \mathrm{p}=0.072)$, prior antibiotics ( $75.4 \%$ vs. $57.6 \%, \mathrm{p}=0.031)$ and $\mathrm{HABSI}(72.3 \%$ vs. $50.0 \%$, $\mathrm{p}=0.009$ ) as risk factors for a HAC than those who were discharged alive.

During the hospitalization and prior to sepsis onset, patients who did not survive had a far longer pre-sepsis median hospital length of stay (LOS) (13 vs. 2 days, $\mathrm{p}=$ 0.002) (Table 3). All markers of severity of acute illness
Table 1 Susceptibilities to additional antimicrobials stratified by carbapenem susceptibility

\begin{tabular}{|c|c|c|c|c|c|c|c|c|}
\hline \multirow[t]{2}{*}{ Drug } & \multicolumn{4}{|c|}{ Carbapenem-S } & \multicolumn{4}{|c|}{ Carbapenem-NS } \\
\hline & $S$ & $\mathbf{R}$ & 1 & NR & $S$ & $\mathbf{R}$ & 1 & NR \\
\hline Cefepime & 31 & 17 & 2 & 0 & 1 & 74 & 1 & 0 \\
\hline Ciprofloxacin & 27 & 17 & 5 & 1 & 0 & 74 & 1 & 1 \\
\hline Gentamycin & 37 & 10 & 2 & 1 & 31 & 34 & 10 & 1 \\
\hline Piperacillin-Tazobactam & 22 & 16 & 9 & 3 & 1 & 71 & 3 & 1 \\
\hline Tobramycin & 19 & 1 & 0 & 30 & 28 & 20 & 8 & 20 \\
\hline Trimethoprim-Sulfamethoxazole & 34 & 13 & 2 & 1 & 8 & 67 & 0 & 1 \\
\hline Amikacin & 3 & 1 & 0 & 46 & 27 & 20 & 5 & 24 \\
\hline Ampicillin-Sulbactam & 0 & 0 & 2 & 48 & 4 & 16 & 19 & 37 \\
\hline Aztreonam & 1 & 0 & 0 & 46 & 41 & 0 & 0 & 35 \\
\hline Colistin & 4 & 0 & 0 & 46 & 53 & 0 & 0 & 23 \\
\hline Minocycline & 2 & 2 & 0 & 46 & 20 & 24 & 8 & 24 \\
\hline Tygecycline & 0 & 2 & 0 & 48 & 5 & 7 & 17 & 47 \\
\hline Doxycycline & 0 & 2 & 0 & 48 & 19 & 19 & 2 & 36 \\
\hline
\end{tabular}

$\mathrm{S}=$ susceptible, NS = non-susceptible, $\mathrm{R}=$ resistant $\mathrm{I}=$ intermediate, $\mathrm{NR}=$ not reported.

were higher in patients who died compared to those who survived; this included the APACHE II score, the presence of septic shock and the need for mechanical ventilation (Table 3). Urine and infected line were less likely and lung was more likely as a source of infection among non-survivors compared to survivors. In contrast to pre-sepsis LOS, post-sepsis onset median LOS was far shorter among those who did not than those who did survive their hospitalization ( 4.5 vs. 15 days, $\mathrm{p}<0.001$ ).

There were substantial differences between the two groups in terms of the likelihood of CRAc as the sepsis culprit $(69.2 \%$ among non-survivors vs. $47.0 \% \%$ among survivors, $\mathrm{p}=0.010$ ) (Table 3). Additionally, non-survivors were approximately $50 \%$ more likely to receive non-IAAT than those patients who survived their hospitalization $(83.1 \%$ vs. $59.1 \%, \mathrm{p}=0.002)$. Notably, among those patients who harbored CRAc, the risk for being treated inappropriately was $96.1 \%$, compared to $36.4 \%$ among those with a susceptible organism, $\mathrm{p}<0.001$.

Table 4 shows the results of a multiple logistic regression model derivation to examine the variables associated with hospital mortality in this population. In this model, receiving non-IAAT was the strongest predictor of hospital death, with the corrected risk ratio of 1.42 (95\% confidence interval 1.10 to $1.58, \mathrm{p}=0.015$ ). A Cox proportional hazards model, confirmed non-IAAT (hazard ratio 2.37, 95\% confidence interval 1.16 to $4.85, \mathrm{p}=0.019$ ) and APACHE II score (hazard ratio 1.08, 95\% confidence interval 1.03 to $1.12, \mathrm{p}<0.001$; per 1 point) as risk factors for hospital death. In an additional logistic regression model to examine factors that contribute to the inappropriate choice of therapy, CRAc as the etiology of sepsis 
Table 2 Baseline characteristics

\begin{tabular}{|c|c|c|c|c|c|}
\hline & Died & $\%$ & Survived & $\%$ & $P$ value \\
\hline & $n=65$ & $49.62 \%$ & $n=66$ & $50.38 \%$ & \\
\hline Age, yrs & & & & & \\
\hline Mean [SD] & $\begin{array}{l}61.9 \\
{[17.4]}\end{array}$ & & $\begin{array}{l}55.2 \\
{[14.7]}\end{array}$ & & \\
\hline Median (IQR) & $\begin{array}{l}63(51, \\
76)\end{array}$ & & $\begin{array}{l}56(45, \\
66)\end{array}$ & & 0.014 \\
\hline Gender, male & 32 & $49.23 \%$ & 32 & $48.48 \%$ & 0.932 \\
\hline Comorbidities & & & & & \\
\hline $\mathrm{CHF}$ & 24 & $36.92 \%$ & 14 & $21.21 \%$ & 0.048 \\
\hline COPD & 23 & $35.38 \%$ & 17 & $25.76 \%$ & 0.232 \\
\hline CLD & 10 & $15.38 \%$ & 3 & $4.55 \%$ & 0.038 \\
\hline DM & 26 & $40.00 \%$ & 12 & $18.18 \%$ & 0.006 \\
\hline CKD & 22 & $33.85 \%$ & 10 & $15.15 \%$ & 0.013 \\
\hline CA & 13 & $20.00 \%$ & 13 & $19.70 \%$ & 0.965 \\
\hline Charlson & & & & & \\
\hline Mean [SD] & $5.3[3.6]$ & & $3.7[3.3]$ & & \\
\hline Median (IQR) & $5(2,8)$ & & $3(1,6)$ & & 0.009 \\
\hline Admission source & & & & & \\
\hline Home & 31 & $47.69 \%$ & 32 & $48.48 \%$ & 0.928 \\
\hline Another hospital & 15 & $23.08 \%$ & 21 & $31.82 \%$ & 0.262 \\
\hline $\mathrm{NH} / \mathrm{ECF}$ & 16 & $24.62 \%$ & 11 & $16.67 \%$ & 0.261 \\
\hline Unknown & 3 & $4.62 \%$ & 2 & $3.03 \%$ & 0.680 \\
\hline HAC RFs & & & & & \\
\hline Dialysis & 18 & $28.12 \%$ & 10 & $15.15 \%$ & 0.072 \\
\hline $\begin{array}{l}\text { Immune } \\
\text { suppression }\end{array}$ & 19 & $29.23 \%$ & 15 & $22.73 \%$ & 0.396 \\
\hline Prior hospitalization & 47 & $72.31 \%$ & 46 & $69.70 \%$ & 0.742 \\
\hline Prior antibiotics & 49 & $75.38 \%$ & 38 & $57.58 \%$ & 0.031 \\
\hline $\mathrm{HABSI}^{\mathrm{a}}$ & 47 & $72.31 \%$ & 33 & $50.00 \%$ & 0.009 \\
\hline
\end{tabular}

$\mathrm{SD}=$ standard deviation; $\mathrm{IQR}=$ interquartile range; $\mathrm{CHF}=$ congestive heart failure; $C O P D=$ chronic obstructive pulmonary disease; $C L D=$ chronic liver disease; $\mathrm{DM}=$ diabetes mellitus; $\mathrm{CKD}=$ chronic kidney disease; $\mathrm{CA}=$ cancer; $\mathrm{NH}=$ nursing home; $\mathrm{ECF}=$ extended care facility; $\mathrm{HCA}=$ healthcare-associated; $\mathrm{RF}=$ risk factors.

${ }^{\mathrm{a}}$ Hospital-acquired BSI defined as BSI that developed after day 2 of hospitalization.

was a strong predictor of inappropriate treatment, with the corrected risk ratio measuring 2.66 (95\% confidence interval 2.43 to $2.72, \mathrm{p}<0.001$ ). The only other predictor retained in the model was congestive heart failure, with the corrected risk ratio 1.89 (95\% confidence interval 1.01 to $2.63, \mathrm{p}=0.048$ ) (AUROC 0.884, Hosmer-Lemeshow $\mathrm{p}=0.513)$.

\section{Discussion}

In the current cohort of patients with sepsis due to Acinetobacter spp., the prevalence of carbapenem resistance was high at nearly $60 \%$. More importantly, the risk for receiving non-IAAT in this setting was extreme.
Table 3 Infection characteristics

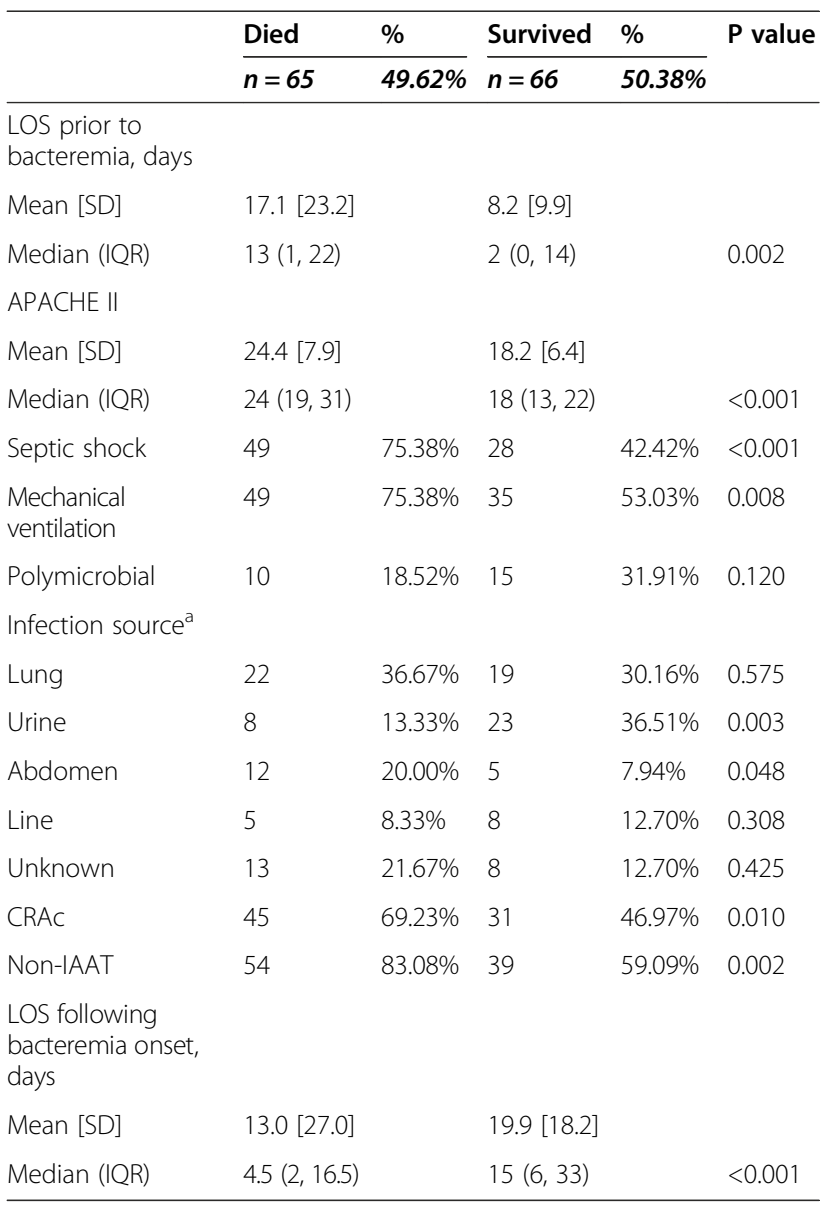

$\mathrm{LOS}=$ length of stay; $\mathrm{SD}=$ standard deviation; $\mathrm{IQR}=$ interquartile range; CRAC = carbapenem-resistant Acinetobacter spp.; IAAT = initially appropriate antibiotic therapy.

aultiple sources possible.

Specifically, the presence of CRAc as the infectious pathogen more than doubled the risk of receiving nonIAAT compared to having a carbapanem-susceptible isolate. Additionally, despite the high baseline rate of death in patients with Acinetobacter spp. sepsis, failure to receive appropriate therapy further increased the risk of hospital mortality.

A vast volume of research has emphasized the importance of early appropriate antimicrobial therapy in the setting of serious infections. Indeed, it has been shown in sepsis and pneumonia that the penalty for the wrong choice of empiric treatment is a 2-4-fold increase in the risk of death [6-8,10-14], and that escalation of treatment in response to culture results fails to mitigate this increase in risk [9]. Our findings generally confirm this relationship. However, the current study adds to these earlier analyses by focusing specifically on a pathogen that is generally only sporadically found in US ICUs. The majority of earlier studies dealing with inappropriate therapy has addressed specific disease states (e.g., pneumonia), irrespective of the 
Table 4 Independent predictors of mortality*

\begin{tabular}{llll}
\hline & $\begin{array}{l}\text { Corrected risk } \\
\text { ratio }\end{array}$ & $\begin{array}{l}\mathbf{9 5 \%} \text { confidence } \\
\text { interval }\end{array}$ & P value \\
\hline Non-IAAT & 1.418 & $1.099-1.583$ & 0.015 \\
APACHE II & 1.056 & $1.025-1.087$ & $<0.001$ \\
$\begin{array}{l}\text { Infection source: } \\
\text { Urine }\end{array}$ & 0.402 & $0.155-0.870$ & 0.018 \\
\hline
\end{tabular}

*Factors excluded from the model for collinearity: chronic kidney disease, hemodialysis, chronic obstructive pulmonary disease, diabetes mellitus (collinear with congestive heart failure); hospital-acquired blood stream infection, LOS prior to the onset of sepsis (collinear with prior antibiotics); Charlson comorbidity score (collinear with age)' mechanical ventilation, vasopressors (collinear with APAHCE II); carbapenem resistance (collinear with non-IAAT).

Factors included but not retained in the model at the $p<0.05$ : congestive heart failure, chronic liver disease, prior antibiotics, age, prior hospitalization, polymicrobial infection, infection sources urine and abdomen.

IAAT = initially appropriate antibiotic therapy.

AUROC $=0.801$, Hosmer-Lemeshow $p=0.406$.

pathogen, or has not attempted to measure impact of inappropriate therapy in the setting of sepsis and septic shock as a syndrome. In this vein, few earlier efforts have dealt specifically with the issue of Acinetobacter spp. as a cause of bacteremia, and no other to the best of our knowledge has examined severe sepsis/septic shock. Indeed, the data on whether non-IAAT in the setting of Acinetobacter bacteremia as a contributor to the increase in the risk of death are conflicting. A small multi-center retrospective matched cohort study from Korea reported that nonIAAT was associated with a 6-fold increase in 30-day mortality [19]. Similarly, a single-center study from Turkey reported a nearly identical risk of 30-day morality to ours in association with non-IAAT (hazard ratio 2.1, 95\% CI 1.2-3.7; $\mathrm{P}=0.007$ ) [20]. In contrast, several other studies failed to detect this relationship, though each suffered from a small sample size of bacteremia cases and other methodologic issues [21-24]. A cohort study from Turkey examining 100 cases of Acinetobacter bacteremia reported that carbapenem resistance was an independent risk factor for 14-day mortality [25]. However, while inappropriate empiric treatment was associated with an increase in mortality in the univariate analysis, it was not reported to be so in a multivariate analysis. It is unclear whether it was included in such and fell out or whether it was simply not examined. In either case, since we have demonstrated strong collinearity of non-IAAT with carbapanem resistance, including both in a single regression would not be statistically desirable. Another small cohort study conducted in the US examining the relationship between carbapenem resistance and mortality in $A$. baumanii bacteremia failed to detect an association, although appropriateness of treatment was an important determinant of hospital death [26]. However, such strong collinearity between non-IAAT and CRAc as we have detected in our study suggests that they may exist in the same causal pathway vis-à-vis outcomes. For this reason, it may be more statistically valid to examine them separately, as we have done, rather than in the same model.

Importantly, none of the above studies focused on the syndrome of sepsis. Because of the populations addressed in and limitations of earlier reports, ours expands upon past research exploring the mortality burden specifically related to Acinetobacter spp. severe sepsis and septic shock. Significantly, we illustrate that although outcomes are generally poor in persons infected with this organism, the additional impact of inappropriate therapy is substantial. As such, this suggests that there is an urgent need for agents that can provide empiric coverage for this pathogen. This last point is even more crucial in that the prevalence of drug-resistant Acinetobacter spp. is increasing both in the US and across the globe [27]. Thus, what may currently be of only a limited burden may in the future become much more of an issue. Conversely, our results emphasize the importance of public policy tools that foster drug development, such as the GAIN act, as well as the Food and Drug Administration's attention to streamlining the development of antibacterial therapies in the setting of unmet medical needs [28].

Our results further suggest that carbapenem resistance is an important risk factor for receiving inappropriate empiric coverage. In other words, the key issue may not only be rapid identification of subjects specifically at risk for Acinetobacter spp., but may in fact be determining whether a patient is suffering from a potentially carbapenemresistant pathogen. This not only highlights the urgent need for concerted efforts at preventing individual infections and curtailing the development and spread of resistant organisms, but also underscores the needs for novel rapid diagnostic tools. Moreover, these new diagnostics must provide clinicians up front with information about a pathogen's likely susceptibilities rather than just simply identifying the organism.

Our study has a number of limitations. As a retrospective cohort it is prone to several forms of bias, most notably selection bias. We attempted to mitigate this by enrolling consecutive patients fitting the pre-determined enrollment criteria. Although we dealt with confounders by adjusting for those that were available, it is possible that some residual confounding remains, particularly confounding by indication. The fact that this is a single-center study in a very specific population of patients (those with Acinetobacter spp. sepsis) may diminish the generalizability of our results to other centers and populations. An additional potential threat to the generalizability of the findings is the fact that non-susceptibility to carbapanems was based on the corresponding year's CLSI threshold for resistance. Applying higher MIC cut-offs either for carbapenems or other agents that have shown at least some in vitro susceptibilities at higher MICs would have reclassified some of the non-IAAT patients into the IAAT category $[29,30]$. 
This reclassification would have the potential either to strengthen or to weaken the association between nonIAAT and mortality. It is important to note that, although our results strongly suggest that the association of carbapenem resistance with an increased risk of death is mechanistically related to the risk of receiving inappropriate empiric therapy, we cannot rule out that resistant Acinetobacter spp. may exert its lethal effect directly by virtue of higher virulence, as has been noted with other pathogens exhibiting higher MICs to certain antimicrobials [31,32]. Because we examined hospital mortality rather than the more standard 28-day mortality as the primary outcome for our study, we may have underestimated the magnitude of this outcome.

\section{Conclusions}

In summary, our study sheds light on the mechanism for the detrimental effect on mortality of exposure to nonIAAT in the setting of Acinetobacter spp. sepsis. Namely, it is the pathogen's resistance to carbapenems, the class of last resort, that appears to mediate at least in part this adverse outcome. In this way, this pathogen is an eloquent illustration of a recent World Health Organization's statement: "A post-antibiotic era - in which common infections and minor injuries can kill - far from being an apocalyptic fantasy, is instead a very real possibility for the 21st Century" [33].

\section{Competing interests}

MDZ has served as a consultant to and/or received research funding from Cubist, Astellas, Pfizer and CareFusion and Theravance. AFS has served as a consultant to and/or received research funding from Cubist, Astellas, Pfizer, Forest, Theravance and CareFusion. STM has served as a consultant to and/or received research funding from Cubist, Astellas and Pfizer. MHK has served as a consultant to and/or received research funding from Cubist, Astellas, Pfizer, Forest and Theravance.

\section{Authors' contributions}

MDZ participated in conception, design, analysis and interpretation of the data, drafted the manuscript and has given final approval for the version to be published. MDZ takes responsibility for data accuracy and analytic and reporting integrity of the study. AFS participated in conception, design, analysis and interpretation of the data. He was involved in revising the manuscript critically for important intellectual content, and has given final approval for the version to be published. STM participated in conception, design, acquisition and interpretation of the data. He was involved in revising the manuscript critically for important intellectual content, and has given final approval for the version to be published. MHK participated in conception, design, acquisition and interpretation of the data. He was involved in revising the manuscript critically for important intellectual content, and has given final approval for the version to be published. All authors read and approved the final manuscript.

\section{Acknowledgements}

This study was supported by a grant from Tetraphase Pharmaceutical, Inc., Watertown, MA. The funder had no role in study design, analyses, data interpretation, or in the preparation or submission of the manuscript. Although the funder was given the opportunity to review the manuscript prior to submission, all of the editorial decisions resided with the authors. No one other than the listed authors contributed to the study.

\section{Disclosure}

This study was supported by a grant from Tetraphase Pharmaceuticals, Inc., Watertown, MA; Dr. Kollef's time was in part supported by the Barnes-Jewish
Hospital Foundation. These data in part have been accepted for presentation at ID Week 2014 meeting in Philadelphia, PA, USA.

\section{Author details}

'Washington Hospital Center, 110 Irving St NW, Washington, DC 20010, USA. ${ }^{2}$ EviMed Research Group, LLC, Goshen, MA, USA. ${ }^{3}$ School of Public Health and Health Sciences, University of Massachusetts, Amherst, MA, USA. ${ }^{4}$ St. Louis College of Pharmacy, 4588 Parkview Place, St. Louis, MO 63110, USA. ${ }^{5}$ Barnes-Jewish Hospital, St. Louis, MO, USA. ${ }^{6}$ Division of Pulmonary and Critical Care Medicine, Washington University School of Medicine, 660 South Euclid Avenue, Campus Box 8052, St. Louis, MO 63110, USA.

Received: 19 August 2014 Accepted: 17 October 2014

Published online: 30 October 2014

\section{References}

1. Centers for Disease Control and Prevention: Antibiotic Resistance Threats in the United States, 2013. Available at http://www.cdc.gov/ drugresistance/threat-report-2013/pdf/ar-threats-2013-508.pdf\#page=59 Accessed May 29, 2014

2. Hidron Al, Edwards JR, Patel J, Horan TC, Sievert DM, Pollock DA, Fridkin SK, National Healthcare Safety Network Team; Participating National Healthcare Safety Network Facilities: NHSN annual update: antimicrobial-resistant pathogens associated with healthcare-associated infections: annual summary of data reported to the National Healthcare Safety Network at the Centers for Disease Control and Prevention, 2006-2007. Infect Control Hosp Epidemiol 2008, 29:996-1011.

3. Sievert DM, Ricks P, Edwards JR, Schneider A, Patel J, Srinivasan A, Kallen A, Limbago B, Fridkin S, National Healthcare Safety Network (NHSN) Team and Participating NHSN Facilities: Antimicrobial-resistant pathogens associated with healthcare-associated infections: Summary of data reported to the National Healthcare Safety Network at the Centers for Disease Control and Prevention, 2009-2010. Infect Control Hosp Epidemiol 2013, 34:1-14.

4. Zilberberg MD, Shorr AF: Prevalence of multidrug-resistant Pseudomonas aeruginosa and carbapenem-resistant Enterobacteriaceae among specimens from hospitalized patients with pneumonia and bloodstream infections in the United States from 2000 to 2009. J Hosp Med 2013, 8:559-563

5. Zilberberg MD, Shorr AF: Secular trends in gram-negative resistance among urinary tract infection hospitalizations in the United States, 2000-2009. Infect Control Hosp Epidemiol 2013, 34:940-946.

6. Micek ST, Kollef KE, Reichley RM, Roubinian N, Kollef MH: Health care-associated pneumonia and community-acquired pneumonia: a single-center experience. Antimicrob Agents Chemother 2007, 51:3568-3573.

7. Iregui M, Ward S, Sherman G, Fraser VJ, Kollef MH: Clinical importance of delays in the initiation of appropriate antibiotic treatment for ventilator-associated pneumonia. Chest 2002, 122:262-268.

8. Alvarez-Lerma F, ICU-acquired Pneumonia Study Group: Modification of empiric antibiotic treatment in patients with pneumonia acquired in the intensive care unit. Intensive Care Med 1996, 22:387-394.

9. Zilberberg MD, Shorr AF, Micek MT, Mody SH, Kollef MH: Antimicrobial therapy escalation and hospital mortality among patients with HCAP: a single center experience. Chest 2008, 134:963-968.

10. Shorr AF, Micek ST, Welch EC, Doherty JA, Reichley RM, Kollef MH: Inappropriate antibiotic therapy in Gram-negative sepsis increases hospital length of stay. Crit Care Med 2011, 39:46-51.

11. Kollef $M H$, Sherman G, Ward S, Fraser VJ: Inadequate antimicrobial treatment of infections: a risk factor for hospital mortality among critically ill patients. Chest 1999, 115:462-474.

12. Garnacho-Montero J, Garcia-Garmendia UL, Barrero-Almodovar A Jimenez-Jimenez FJ, Perez-Paredes C, Ortiz-Leyba C: Impact of adequate empirical antibiotic therapy on the outcome of patients admitted to the intensive care unit with sepsis. Crit Care Med 2003, 31:2742-2751.

13. Harbarth S, Garbino J, Pugin J, Romand JA, Lew D, Pittet D: Inappropriate initial antimicrobial therapy and its effect on survival in a clinical trial of immunomodulating therapy for severe sepsis. Am J Med 2003, 115:529-535.

14. Kumar A, Roberts D, Wood KE, Light B, Parrillo JE, Sharma S, Suppes R, Feinstein D, Zanotti S, Taiberg L, Gurka D, Kumar A, Cheang M: Duration of hypotension before initiation of effective antimicrobial therapy is the critical determinant of survival in human septic shock. Crit Care Med 2006, 34:1589-1596. 
15. Dellinger RP, Levy MM, Rhodes A, Annane D, Gerlach H, Opal SM, Sevransky JE, Sprung CL, Douglas IS, Jaeschke R, Osborn TM, Nunnally ME, Townsend SR, Reinhart K, Kleinpell RM, Angus DC, Deutschman CS, Machado FR, Rubenfeld GD, Webb SA, Beale RJ, Vincent IL, Moreno R, Surviving Sepsis Campaign Guidelines Committee including the Pediatric Subgroup: Surviving Sepsis Campaign: international guidelines for management of severe sepsis and septic shock: 2012. Crit Care Med 2013, 41:580-637.

16. Angus DC, Linde-Zwirble WT, Lidicker J, Clermont G, Carcillo J, Pinsky MR: Epidemiology of severe sepsis in the United States: analysis of incidence, outcome, and associated costs of care. Crit Care Med 2001, 29:1303-1310.

17. Knaus WA, Draper EA, Wagner DP, Zimmerman JE: APACHE II: a severity of disease classification system. Crit Care Med 1985, 13:818-829.

18. Zhang J, Kai YF: What's the Relative Risk?: A Method of Correcting the Odds Ratio in Cohort Studies of Common Outcomes. JAMA 1998, 280:1690-1691.

19. Kwon KT, Oh WS, Song JH, Chang HH, Jung SI, Kim SW, Ryu SY, Heo ST, Jung DS, Rhee JY, Shin SY, Ko KS, Peck KR, Lee NY: Impact of imipenem resistance on mortality in patients with Acinetobacter bacteraemia. $J$ Antimicrob Chemother 2007, 59:525-530.

20. Erbay A, Idil A, Gozel MG, Mumcuoglu I, Balaban N: Impact of early appropriate antimicrobial therapy on survival in Acinetobacter baumannil bloodstream infections. Int J Antimicrob Agnets 2009, 34:575-579.

21. Sunenshine RH, Wright MO, Maragakis LL, Harris AD, Song X, Hebden J, Cosgrove SE, Anderson A, Carnell J, Jernigan DB, Kleinbaum DG, Perl TM, Standiford HC, Srinivasan A: Multidrug-resistant Acinetobacter infection mortality rate and length of hospitalization. Emerg Infect Dis 2007, 13:97-103.

22. Chen HP, Chen TL, Lai CH, Fung CP, Wong WW, Yu KW, Liu CY: Predictors of mortality in Acinetobacter baumannii bacteremia. J Microbiol Immunol Infect 2005, 38:127-136.

23. Choi JY, Park YS, Kim CO, Yoon HJ, Shin SY, Kim YA, Song YG, Yong D, Lee K, Kim JM: Mortality risk factors of Acinetobacter baumannii bacteraemia. Intern Med J 2005, 35:599-603.

24. Falagas ME, Kasiakou SK, Rafailidis PI, Zouglakis G, Morfou P: Comparison of mortality of patients with Acinetobacter baumannii bacteraemia receiving appropriate and inappropriate empirical therapy. J Antimicrob Chemother 2006, 57:1251-1254.

25. Metan G, Sariguzel F, Sumerkan B: Factors influencing survival in patients with multi-drug-resistant Acinetobacter bacteraemia. Eur J Intern Med 2009, 20:540-544.

26. Esterly JS, Griffith M, Qi C, Manczynski M, Postelnick MJ, Scheetz MH: Impact of carbapenem resistance and receipt of active antimicrobial therapy on clinical outcomes of Acinetobacter baumannii bloodstream infections. Antimicrob Agents Chemother 2011, 55:4844-4849.

27. Maragakis LL, Perl TM: Acinetobacter baumannii: epidemiology, antimicrobial resistance, and treatment options. Clin Infect Dis 2008, 46:1254-1263.

28. US Department of Health and Human Services, Food and Drug Administration, Center for Drug Evaluation and Research: Guidance for Industry: Antibacterial Therapies for Patients With Unmet Medical Need for the Treatment of Serious Bacterial Diseases. 2013. Available at http:// www.fda.gov/downloads/drugs/guidancecomplianceregulatoryinformation/ guidances/ucm359184.pdf; accessed June 18, 2014

29. Kiratisin P, Apisarnthanarak A, Kaewdaeng S: Synergistic activities between carbapenems and other antimicrobial agents against Acinetobacter baumannii including multidrug-resistant and extensively drug-resistant isolates. Int J Antimicrob Agents 2010, 36:243-246.

30. Sheng WH, Wang JT, Li SY, Lin YC, Cheng A, Chen YC, Chang SC: Comparative in vitro antimicrobial susceptibilities and synergistic activities of antimicrobial combinations against carbapenem-resistant Acinetobacter species: Acinetobacter baumannii versus Acinetobacter genospecies 3 and 13TU. Diagn Microbiol Infect Dis 2011, 70:380-386.

31. Holmes NE, Turnidge JD, Munckhof WJ, Robinson JO, Korman TM, O'Sullivan MV, Anderson TL, Roberts SA, Gao W, Christiansen K, Coombs GW, Johnson PD, Howden BP: Antibiotic choice may not explain poorer outcomes in patients with Staphylococcus aureus bacteremia and high vancomycin minimum inhibitory concentrations. J Infect Dis 2011, 204:340-347.
32. Cervera C, Castaneda X, de la Maria CG, del Rio A, Moreno A, Soy D, Pericas JM, Falces C, Armero Y, Almela M, Ninot S, Pare JC, Mestres CA, Gatell JM, Marco F, Miro JM, Hospital Clinic Endocarditis Study Group: Effect of vancomycin minimal inhibitory concentration on the outcome of methicillin-susceptible Staphylococcus aureus endocarditis. Clin Infect Dis 2014, 58:1668-1675.

33. World Health Organization: Antimicrobial resistance: Global report on surveillance 2014. Available at URL: Please check that the following URLS are working. If not, please provide alternatives: http://www.who.int/ drugresistance/documents/surveillancereport/en/; accessed June 5, 2014.

\section{doi:10.1186/s12879-014-0572-6}

Cite this article as: Shorr et al:: Predictors of hospital mortality among septic ICU patients with Acinetobacter spp. bacteremia: a cohort study. BMC Infectious Diseases 2014 14:572.

\section{Submit your next manuscript to BioMed Central and take full advantage of:}

- Convenient online submission

- Thorough peer review

- No space constraints or color figure charges

- Immediate publication on acceptance

- Inclusion in PubMed, CAS, Scopus and Google Scholar

- Research which is freely available for redistribution

Submit your manuscript at www.biomedcentral.com/submit
C) BioMed Central 